TITLE:

RECORD OF AN UNUSUAL FISH

STRANDING IN WINTER, WITH THE

LIST OF STRANDED FISHES

IDENTIFIED BY PROF. K.

MATSUBARA

$\operatorname{AUTHOR}(\mathrm{S})$ :

Tokioka, Takasi

CITATION:

Tokioka, Takasi. RECORD OF AN UNUSUAL FISH STRANDING IN WINTER, WITH THE LIST OF STRANDED FISHES IDENTIFIED BY PROF. K. MATSUBARA. PUBLICATIONS OF THE SETO MARINE BIOLOGICAL LABORATORY 1961, 9(2): 447-450

ISSUE DATE:

1961-12-20

URL:

http://hdl.handle.net/2433/175283

RIGHT: 


\title{
RECORD OF AN UNUSUAL FISH STRANDING IN WINTER, WITH THE LIST OF STRANDED FISHES IDENTIFIED \\ BY PROF. K. MATSUBARA
}

\author{
TAKASI TOKIOKA
}

Seto Marine Biological Laboratory

With two Tables

Cold weather had continued for several days in the middle of February of this year and on the morning of the nineteenth, Sunday, I was alarmed by my boy, TAKasi junior, who was much amazed at abundant fishes stranded on the beach near the laboratory on the way of his daily walk with his dog. It was very cold for this region; the thermometer stood at 0.2 Centigrade. He brought one of those fishes with him and I found it was Fistularia petimba LACÉPÈDE. Thinking that those fishes might be good as food for dog, my boy and I went down to the beach north of the laboratory and began to pick up fishes. At first, being interested in picking up fishes, I continued collecting, but soon I was impressed by so many kinds of those stranded fishes and changed my mind to continue collecting to examine fishes closely and record the event of the morning exactly, but not to secure much food for our dog. Now, my girl, Mié, was also called out for help and after an hour our buckets became enough heavy when we finished collecting along the beach of about $200 \mathrm{~m}$ long.

Being assisted by all hands of the family, the collected specimens were washed and sorted carefully. For most species, several of each species were sent to Prof. K. Matsubara of the Fisheries Institute of Kyoto University for examination, who identified them and gave me the list of fishes he examined. I want to express here my hearty thanks for his kindness in doing such things for me.

In all, about 1600 fishes belonging to 51 different species were included in the collection. Important specimens were presented to the ichthyological laboratory of the Fisheries Institute of Kyoto University and some to the Osaka Museum of Natural History, while others were tasted by my family members together with Mr. Genjiro FUKUDA, the president of the publishing company known by the Illustrated Encyclopedia of the Fauna of Japan, or served as food for dog.

1) Contributions from the Seto Marine Biological Laboratory, No. 377.

Publ. Seto Mar. Biol. Lab., IX (2), 1961. (Article 23) 
Table 1. List of stranded fishes.

Name

1. Pterophryne histrio (LINNÉ)

$$
\text { (ハナオコゼ) }
$$

2. Antennarius sanguifluus JORDAN (ベニイザリウオ)

3. Diodon holacanthus LINNÉ

$$
\text { (ハリセンボン) }
$$

4. Fugu niphobles (JORDAN \& SNYDER) (クサフグ)

5. Canthigaster rivulatus (TEMMINCK \& SCHLEGEL) (キタマクラ)

6. Canthigaster valentini (BLEEKER) (シマキンチヤクフグ)

7. Lactoria fornasini (BIANCONI) (シマウミスズメ)

8. Lactoria diaphanus (BLOCH \& SCHNEIDER) (ウミスズメ)

9. Apistus carinatus (BLOCH \& SCHNEIDER)

$$
\text { (八千) }
$$

10. Pterois lunulata TEMmINCK \& SCHLEgeL (ミノカサゴ)

11. Palachaeturichthys polynema (BLEEKER) (ヒゲハゼ)

12. Ptereleotris microlepis sakurai (SCHMIDT) (サクライクロユリハゼ)

13. Vireosa hanai JORDAN \& SNYDER (ハナハゼ)

14. Aspidontus maroubrae (OGILBY) (クロスジギンポ)

15. Chaetodon auriga FORSKÀI

(トゲチョウチョウウオ）

16. Pseudolabrus japonicus (HoUTTUYN) （ササノハベラ）

17. Chromis notatus (TEMMINCK \& SCHLEGEL) (スズメダイ)

18. Pomacentrus coelestis JORDAN \& STARKS (ソラスズメダイ)

19. Chrysiptera assimilis (GÜNTHER) (ルリスズメ)

20. Amphiprion xanthurus (CUVIER) (クマノミ)

21. Champsodon snyderi FRANZ (ワニギス)

22. Brotula multibarbata TEMMINCK \& SCHLEGEL (イタチウオ)

23. Jordanicus sagamianus (TANAKA) （カクレウオ）

24. Leptoscolopsis nagasakiensis TANAKA (イトタマガシラ)

25. Franzia squamipinnis (PETERS) (キンギヨハナダイ)
Maximum length Number

$13 \mathrm{~cm}$

1

$8.5 \mathrm{~cm} \quad 1$

1

$12 \mathrm{~cm}$

$11 \mathrm{~cm} \quad 5(0.3 \%)$

$10 \mathrm{~cm} \quad 46(2.9 \%)$

$8 \mathrm{~cm}$

$7(0.4 \%)$

$12 \mathrm{~cm} \quad 1$

$10 \mathrm{~cm}$

$20 \mathrm{~cm} \quad 3(0.2 \%)$

$10 \mathrm{~cm}$

$6 \mathrm{~cm} \quad 71(4.4 \%)$

$4.5 \mathrm{~cm}$

$4 \mathrm{~cm}$

$6 \mathrm{~cm}$

$4 \mathrm{~cm} \quad 6(0.4 \%)$

$10 \mathrm{~cm} \quad 25(1.6 \%)$

$6.5 \mathrm{~cm} \quad 5(0.3 \%)$

$4.5 \mathrm{~cm} \quad, \quad 22(1.4 \%)$

$9 \mathrm{~cm} \quad 6(0.4 \%)$

$4 \mathrm{~cm}$

$31 \mathrm{~cm}$

1

$6 \mathrm{~cm} \quad 1$

$4.5 \mathrm{~cm} \quad 2(0.1 \%)$ 


\section{Name}

26. Grammistes sexlineatus proerythraeus FowLER (ベニヌ)サラシ)

27. Epinephelus megachir (RICHARDSON) (モヨウハタ)

28. Epinephelus fasciatus fasciatus (FORSKÅL) (アカ八夕)

29. *Acropoma japonicum GüNTHER （ホタルジヤコ）

30. Apogon niger DöDERLEIN (クロイシモチ)

31. *Apogon eliotti DAY (ソマグロイシモチ，シチセンイシモチ)

32. Apogon teniatus CUVIER (ヨコスジイシモチ)

33. Apogon schlegeli BLEEKER (コスジイシモチ)

34. Apogon döderleini JORDAN \& SNYDER (オオスジイシモチ)

35. Apogon kiensis JORDAN \& SNYDER (テツポウイシモチ)

36. Apogon semilineatus TEMMINCK \& SCHLEGEL (ネンブツダイ)

37. Apogon notatus (HoUTTUYN) (クロホシイシモ千)

38. Apogon erythrinus kominatoensis EBINA (コミナトイシモチ)

39. Pempheris japonicus DöDERLEIN (八タンポ)

40. Leiognathus elongatus SMITH \& POPE (ヒメヒイラギ)

41. Holocentrus spimo-sisimus TEMMINCK \& SCHLEGEL (イツトウダイ, カノコウオ)

42. *Paratrachichthys prosthemius JORDAN \& FowLER (ハリダシエビス)

43. Fistularia petimba LACÉPÈDE (アカヤガラ)

44. Gymnothrax hepatica (TEMMINCK \& ScHLEGEL) (ヘリシロウツボ)

45. Leiuranus semicinctus (LAY \& BENNETT) (ソラウミヘビ)

46. Anago anago (TEMmincK \& SCHLEGEL) (ゴテンアナゴ)

47. Conger cinereus RÜPPELL (クロアナゴ)

48. Rhynchocymba nystromi nystromi JORDAN \& SNYDER (ギンアナゴ)

49. Uroconger lepturus (RICHARDSON) (アイアナゴ)

50. Echelus uropterus (TEMMINCK \& SCHLEGEL) (ヒレアナゴ)

51. Plotosus anguillaris LACÉPÈDE (ゴンズイ)

\begin{tabular}{|c|c|}
\hline $\begin{array}{l}\text { Maximum length } \\
6 \mathrm{~cm}\end{array}$ & $\begin{array}{l}\text { Number } \\
1\end{array}$ \\
\hline $7 \mathrm{~cm}$ & $11(0.7 \%)$ \\
\hline $7 \mathrm{~cm}$ & 1 \\
\hline $5.5 \mathrm{~cm}$ & $37(2.3 \%)$ \\
\hline $9 \mathrm{~cm}$ & $213(13.2 \%)$ \\
\hline $10 \mathrm{~cm}$ & $18(1.1 \%)$ \\
\hline $12 \mathrm{~cm}$ & $50(3.1 \%)$ \\
\hline $9.5 \mathrm{~cm}$ & $669(41.6 \%)$ \\
\hline $9.5 \mathrm{~cm}$ & $30(1.9 \%)$ \\
\hline $5.5 \mathrm{~cm}$ & $5(0.3 \%)$ \\
\hline $8 \mathrm{~cm}$ & $7(0.4 \%)$ \\
\hline $8.5 \mathrm{~cm}$ & $167(10.4 \%)$ \\
\hline $4.5 \mathrm{~cm}$ & $11(0.7 \%)$ \\
\hline $\begin{array}{l}4.2 \mathrm{~cm} \text { (total length) } \\
4.7 \mathrm{~cm} \text { (head length) }\end{array}$ & $3(0.2 \%)$ \\
\hline $4.4 \mathrm{~cm}$ & 1 \\
\hline $11 \mathrm{~cm}$ & 1 \\
\hline $5.5 \mathrm{~cm}$ & 1 \\
\hline \multirow[t]{3}{*}{$95 \mathrm{~cm}$} & $153(9.5 \%)$ \\
\hline & 1 \\
\hline & (on Feb. 20) \\
\hline \multirow[t]{6}{*}{$22 \mathrm{~cm}$} & 1 \\
\hline & 1 \\
\hline & $2(0.1 \%)$ \\
\hline & 1 \\
\hline & 1 \\
\hline & $10(0.6 \%)$ \\
\hline
\end{tabular}

1609 in total

* with luminous organ. 
As I had noticed that a large angler fish, Antennarius scriptissimus Jordan, and a kind of porcupine fishes, Chilomycterus affinis GüNTHER, had been stranded on the same beach since a few days before, it is clear that those fishes were killed by cold and gathered there by gentle north west wind on that Sunday morning.

Table 2. Water temperature in the middle of February, 1961.

\begin{tabular}{|c|c||c|c|}
\hline Date & $\mathrm{t}^{\circ} \mathrm{C}$ & Date & $\mathrm{t}^{\circ} \mathrm{C}$ \\
\hline 12 & 13.48 & 16 & 11.50 \\
13 & 13.00 & 17 & 11.69 \\
14 & 12.59 & 18 & 11.59 \\
15 & 11.00 & 19 & 11.48 \\
\hline
\end{tabular}

